\title{
Sensitivity-enhanced fiber interferometric high temperature sensor based on Vernier effect
}

X. Q. Lei, X. P. Dong

X. Q. Lei, X. P. Dong, "Sensitivity-enhanced fiber interferometric high temperature sensor based on Vernier effect," Proc. SPIE 11144, Photonics and Education in Measurement Science 2019, 1114405 (17 September 2019); doi: $10.1117 / 12.2530864$

SPIE Event: Joint TC1 - TC2 International Symposium on Photonics and Education in Measurement Science 2019, 2019, Jena, Germany 


\title{
Sensitivity-enhanced fiber interferometric high temperature sensor based on Vernier effect
}

\author{
X.Q.Lei ${ }^{1}$, X.P. Dong ${ }^{1,2 *}$ \\ 1. The Institute of Lightwave Technology, School of Electronic Science and Engineering, Xiamen \\ University, Xiamen, Fujian 361005, China \\ 2. Center of Optical Fiber Sensor Technology, Xiamen University Tan Kah Kee College, Zhangzhou, \\ China
}

\begin{abstract}
A novel sensitivity-enhanced intrinsic fiber Fabry-Perot interferometer (IFFPI) high temperature sensor based on a hollow-core photonic crystal fiber (HC-PCF) and modified Vernier effect is proposed and experimentally demonstrated. The all fiber IFFPIs are easily constructed by splicing one end of the HC-PCF to a leading single mode fiber (SMF) and applying an arc at the other end of the HC-PCF to form a pure silica tip. The modified Vernier effect is formed by three beams of lights reflected from the SMF-PCF splicing joint, and the two air/glass interfaces on the ends of the collapsed HC-PCF tip, respectively. Vernier effect was first applied to high temperature sensing up to $1200^{\circ} \mathrm{C}$, in this work, and the experimental results exhibit good stability and repeatability. The temperature sensitivity, measured from the spectrum envelope, is 14 to 57 times higher than that of other configurations using similar HC-PCFs without the Vernier effect. The proposed sensor has the advantages of high sensitivity, good stability, compactness, ease of fabrication, and has potential application in practical high-temperature measurements.
\end{abstract}

Keywords: fiber sensor, Fabry-Perot, interferometer, Vernier effect, dual-cavity, envelope, high temperature measurement, Photonic crystal fiber(PCF), all-optical devices

\section{INTRODUCTION}

High temperature sensing has a wide range of applications in many fields such as metallurgy, energy, and chemical industries[1]. In contrast to their electronic counterparts, fiber-optic temperature sensors exhibit outstanding advantages, such as low-cost, fast response, immunity to electromagnetic interference, and durability in harsh environments [2]. Using conventional step-index fibers, the maximum temperature measurement may be limited in the doped ions (e.g., ions of germanium, phosphorus, and boron, etc.) inside the core of the fiber [3]-[6]. Compared with traditional coredoped fibers, photonic crystal fibers ( $\mathrm{PCFs}$ ), made from pure silica glass (un-doped single-material), which can withstand temperatures over $1000^{\circ} \mathrm{C}$ and has good durability, have been studied intensively [2], [7]-[9].

Vernier effect is an efficient way to improve the accuracy of measurement based on interferometer, has been widely used in calipers and barometers. Recently, several measurement schemes, based on the Vernier effect produced by optical fiber cavities, has been proposed to improve sensitivity by combining paralleled or cascaded interferometers with a small optical path different (OPD). Such as cascaded two Sagnac loops [10], two Fabry-Perot (FP) cavities [11], two offset spliced Mach-Zehnder interferometers (MZIs) [12] and hybrid cascaded configuration constructed by angle shift splicing two segments of polarization maintaining fibers in a single Sagnac loop [13], or combining a FP cavity with a Sagnac loop [14], etc. In the above works, by tracing the envelope of the transmission spectrum, the sensitivity of the Vernier effect-based fiber sensors is effectively improved by several to dozens of times over that using single individual interferometers. However, these traditional multipath interfering configurations generally have shortages of complexity and bulky structures, especially in the Sagnac loops configuration. In the case of in-line cascaded MZIs or FP cavities, because the two cavities may have almost the same response to a change in temperature, one of the two cascaded interferometers must be well shielded to increase the measurement sensitivity, which causes complexity and difficulty in building a compact sensor head.

As reported [15] and [16], a cascaded intrinsic fiber Fabry-Perot interferometers sensor, based on the Vernier effect, is formed in an ultra-compact structure. However, the open cavity structure may cause problems in a high temperature 
sensing environment, because the FP cavities may suffer from disturbances from burning contaminants, which lead to changes in cavity properties. As stated in [3], a section of hollow-core fiber is cascaded by a piece of SMF, and, based on the modified Vernier effect, an amplification factor as high as 133.29 is obtained. However, the maximum temperature of $1050^{\circ} \mathrm{C}$ is limited by the doped core of the conventional SMF used in the experiments. Proposed in [17] is a sphereended PCF structure for high temperature sensing in the absence of Vernier effect, Because the OPD between the cavities is too large.

We propose a novel miniaturized intrinsic fiber Fabry-Perot interferometers (IFFPI) sensor with improved sensitivity, based on the Vernier effect. The sensor is fabricated simply by splicing one end of a section of hollow-core photonic crystal fiber (HC-PCF) to SMF and heating the other end of the HC-PCF, by arc discharging, to form a tiny section of solid collapsed PCF. By tracing the shift of the envelope in the reflection spectrum, the temperature sensitivity is improved significantly. The maximum sensitivity, $15.68 \mathrm{pm} /{ }^{\circ} \mathrm{C}$, with a linearity of $99.85 \%$ in the high temperature range from $300^{\circ} \mathrm{C}$ to $1200^{\circ} \mathrm{C}$, is 14 to 57 times higher than with other configurations using similar HC-PCFs without the Vernier effect [18]-[21]. The sensitivities obtained in our experiments are also higher than those obtained with other compact types of optical fiber temperature sensors [6], [7], [22], [23].

\section{SENSOR STRUCTURE AND PRINCIPLE OF MEASUREMENT}

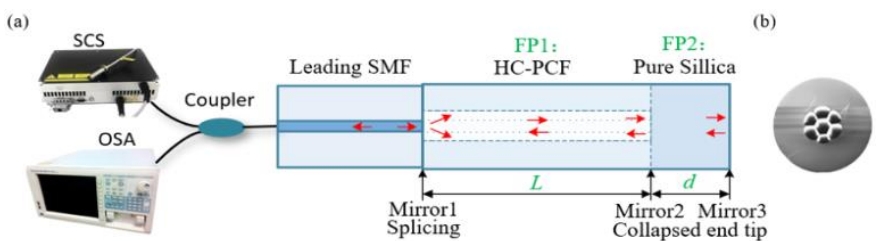

Fig.1. Schematic diagram of the HC-PCF based fiber sensor.(a) Schematic of proposed sensor structure.

(b) Microscope images of the cross section of the HC-PCF

The configuration of a HC-PCF based all-fiber IFFPIs sensor probe is shown in Fig. 1(a). The HC-PCF used in our experiment is fabricated by the company of Yangtze Optical Fiber, Wuhan, China, and SEM photo of the cross-section of this fiber is shown in Fig. 1(b). The HC-PCF has a large hexagonal air core with six surrounding air holes. Because of the antiresonance between the core mode and the modes of the inner silica wall, light can be guided in the air core [24]. The diameters of the hollow core and the air-hole cladding are approximately $15 \mu \mathrm{m}$ and $50 \mu \mathrm{m}$, respectively. The outer diameter of HC-PCF is about $129 \mu \mathrm{m}$. The sensor is fabricated simply by splicing one end of a section of hollow-core photonic crystal fiber (HC-PCF) to a leading SMF and applying an arc at the other hand. When the light from the super continuum laser Source (SC-5, YSL co., Ltd. 480nm-2400nm) is injected into the proposed sensor head through the leading SMF, the so-called modified Vernier effect is formed by three beams of light reflected from one splicing joint and two air/glass interfaces of the pure silica tip: Mirror1, Mirror2, and Mirror3, respectively. Mirror1 and Mirror2 form a closed air FP1, with a length of L. Mirror2 and Mirror3 form a silica FP2, with a length of d. Mirror1 and Mirror3 form an FP3, whose OPD is close toFP1, with a length of $\mathrm{L}+\mathrm{d}$. The resonance modes of these three FP cavities determine the reflection interference spectrum. The power reflection coefficients at Mirror1, Mirror2, and Mirror3 are $R_{1}, R_{2}$, and $R_{3}$, respectively and are defined by the following equations:

$$
\mathrm{R}_{1}=\left|\frac{n_{S M F}-n_{H C P C F}}{n_{S M F}+n_{H C P C F}}\right|^{2}, \mathrm{R}_{2}=\mathrm{R}_{3}=\left|\frac{n_{\text {silica }}-n_{\text {air }}}{n_{\text {silica }}+n_{\text {air }}}\right|^{2}
$$

where, $n_{H C P C F}$ and $n_{\text {silica }}$ are the effective refractive indices of FP1 and FP2, respectively; $n_{\text {air }}$ and $n_{S M F}$ represents the refractive index of air and fiber core of SMF, respectively.

The specific preparation of the proposed sensor is as follows: (1) The coating of the sensing fiber is removed, and the surface of the sensing section is cleaned with anhydrous alcohol. (2) A section of the pure silica HC-PCF is spliced with the leading SMF (Corning SMF-28) by the fusion splicer (DVP-720A). With appropriate discharge time and fusion power $(0.2 \mathrm{~ms}$ and 4 bit,respectively), the splice between the SMF and HC-PCF is made with minimal collapse of the air holes.(3)With the cleaver (Fujikura CT-30), the spliced HC-PCF is terminated at a certain length, L. (4) Subsequently, the cleaved fiber end of the HC-PCF is applied with a slight arc discharge. Air holes in the collapsed portion of the HCPCF are all sealed by the arc discharge processor. Then, a section of the pure silica tipis formed. Compared with other common IFFPI preparation technologies[3], [15], [16], no additional process is required to splice the third fiber and terminate it at a certain length to form the FP2. Instead, applying a slight arc to the end of HC-PCF effectively simplifies 
fabrication of the sensor and formation of a pure silica micro-cavity. To interpret the working principle of the proposed high sensitivity IFFPIs based on the Vernier effect, the whole reflected electric fields $\left(E_{r}\right)$ is calculated as follows[11], [25]:

$$
E_{r}=E_{i n}\left[\sqrt{R_{2}}+A e^{-j 2 \phi_{1}}+B e^{-j 2\left(\phi_{1}+\phi_{2}\right)}\right]
$$

where, $A=\left(1-\varepsilon_{1}\right)\left(1-R_{1}\right) \sqrt{\mathrm{r}_{2}}$, and $B=\left(1-\varepsilon_{1}\right)\left(1-\varepsilon_{2}\right)\left(1-R_{1}\right)\left(1-R_{2}\right) \sqrt{\mathrm{r}_{3}}$; In the above equation, $E_{\text {in }}$ is the input field; $\varepsilon_{1}, \varepsilon_{2}$ are the transmission losses in Mirror1 and Mirror2, respectively; $R_{1}, R_{2}$, and $\mathrm{R}_{3}$ are the reflection coefficients of three mirrors, respectively; $\phi_{1}=2 \pi n_{1} L_{1} / \lambda, \phi_{2}=2 \pi n_{2} L_{2} / \lambda$, are the phase shifts in resonantcavity 1 and cavity 2 , respectively; $n_{1}, n_{2}, L_{1}, L_{2}$ are the effective RI, the length of resonant cavity 1 and cavity 2 , respectively; and $\lambda$ is the wavelength of the input light. The reflection spectrum function is derived from Eq. (2) as follow:

$$
I_{r}=\left(\frac{E_{r}}{E_{\text {in }}}\right)^{2}=R_{1}+A^{2}+B^{2}+2 \sqrt{R_{1}} A \cos \left(2 \phi_{1}\right)+2 A B \cos \left(2 \phi_{2}\right)+2 \sqrt{R_{1}} B \cos \left[2\left(\phi_{1}+\phi_{2}\right)\right]
$$

Fig. 2. Transmission spectra of the proposed sensors obtained from experiment.

Obtained from the experiments are the transmission spectra of the proposed sensors (See Fig. 2). The so-called envelope is obtained by connecting the valleys of the high frequency fringes; for example, the lower envelopes are indicated by the red curves in Fig.2. The same results are achieved by connecting the peaks of the high-frequency fringes. The period of envelope, also called as free spectral range of envelope, $\left(\mathrm{FSR}_{\text {envelope }}\right)$, is given by

$$
\mathrm{FSR}_{\text {envelope }}=\frac{\mathrm{FSR}_{\text {ref }} \mathrm{FSR}_{\text {sens }}}{\mathrm{FSR}_{\text {ref }}-\mathrm{FSR}_{\text {sens }}}
$$

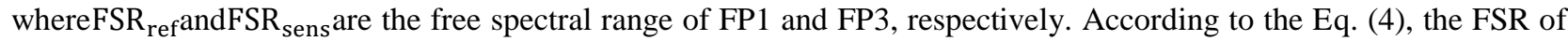
the envelope can be further increased by reducing the difference in period between the two cavities. Therefore, to generate the Vernier effect and improve the accuracy of the measurements by tracing envelope, the OPD of FP3 is set near to, but not equal to that of FP1. Moreover, because of the superposition of the two individual FP cavities, the difference in length between the two resonant cavities is equal to the length of solid tip, d. Therefore, the OPD between the two cavities can be obtained easily by simply arc discharge, and the complexity and costs in fabrication are reduced considerably. The envelope is derived from $\partial \mathrm{I}_{r} / \partial \lambda=0$; then, combing with Eq.(2), the corresponding envelope function is simplified as follows:

$$
F_{c}=2 m \cos \left[4 \pi\left(S_{1}-S_{2}\right) / \lambda\right]
$$

where $\mathrm{m}, S_{1}=\mathrm{n}_{\mathrm{HCPCF}} L$ and $S_{2}=\mathrm{n}_{\mathrm{HCPCF}} L+\mathrm{n}_{\text {silica }} d$ are the amplitude of the envelope, and the optical path of FP1 and FP3, respectively. When the ambient temperature increases, $\phi_{i}, i=1,2$ increases due to the thermo-optic effect and thermal expansion of SHCF and the solid tip, which lead to the shift of the resonant wavelengths of FP1 and FP3. The overlapping portion in FP3 and FP1 shows a relative low sensitivity for temperature sensing because of a low thermooptic effect in the air cavity, which functions as a fixed part of the Vernier-scale. The resonant wavelength of FP3 shifts to a longer wavelength with increasing ambient temperature, because of the positive thermo-optical coefficient and linear thermal expansion coefficient of the silica tip. As a consequence, the envelope has a red shift (See Fig.2), and the amplification factor of the cascaded FPI sensor is expressed as follow: 


$$
\mathrm{M}=\frac{\mathrm{n}_{\mathrm{HCPCF}} L+\mathrm{n}_{\text {silica }} d}{\mathrm{n}_{\text {silica }} d}
$$

Therefore, by tracing the shift of the envelope, the value of the wavelength shift is magnified by an amplification factor compared with the tracing shift of the resonant wavelength of a single FP3. The amplification factor $\mathrm{M}=$ $\mathrm{FSR}_{\text {envelope }} / \mathrm{FSR}_{\text {sens }}$, corresponds with the magnification of the period. Thus, by substituting the corresponding observed value into the Eq. (4), $\mathrm{M}$ of the proposed sensor is approximately 6.99,which agrees well with Eq.(6). Therefore, the Vernier effect significantly improves the sensitivity of the sensor. As indicated by Eq. (6), the amplification factor could be further increased by reducing the length of the silica fiber tip and increasing the length of the HC-PCF cavity.

\section{EXPERIMENTAL RESULTS AND DISCUSSION}

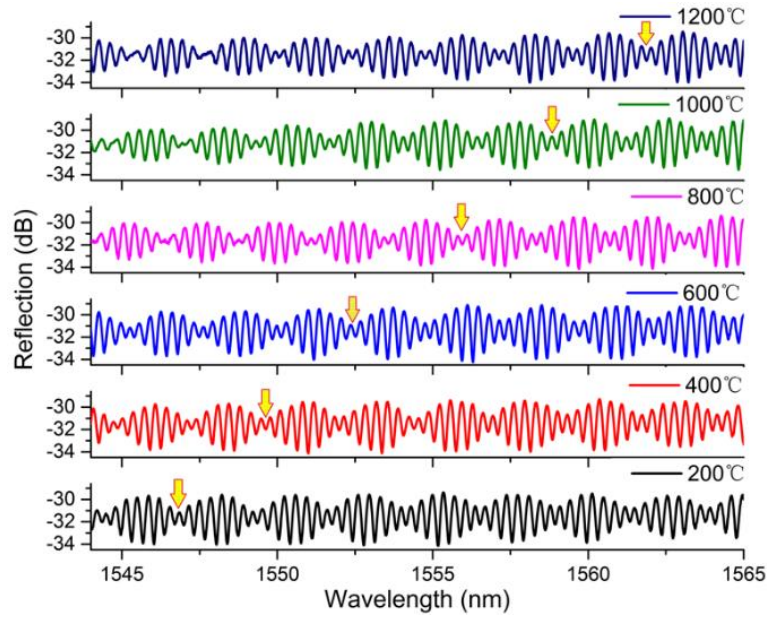

Fig.3.Reflection spectra of proposed sensor under different temperature varying from 200 to $1200^{\circ} \mathrm{C}$.

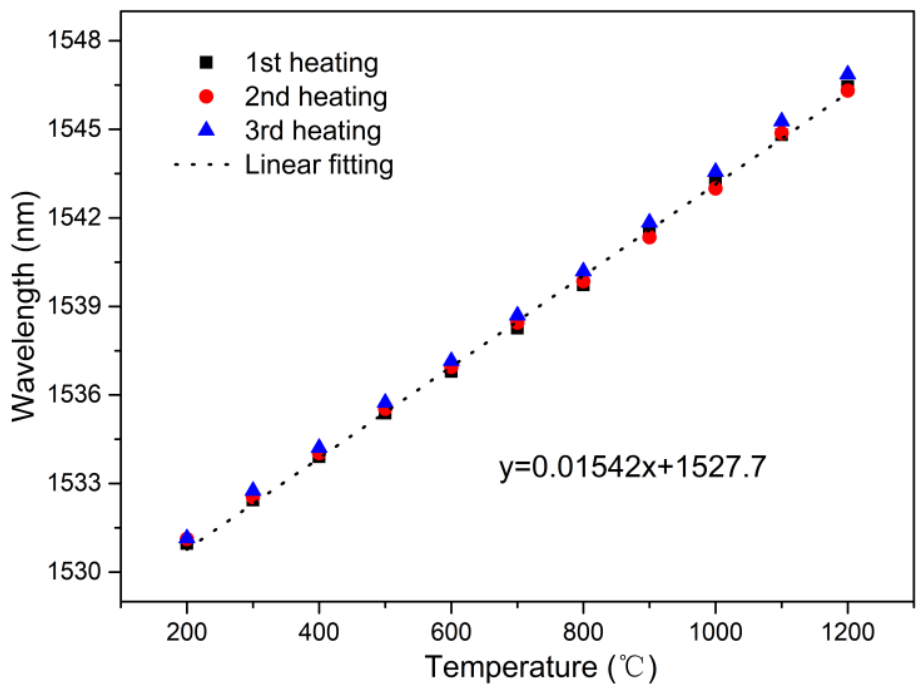

Fig.4. The fitting-figures of the relationship between wavelength drift and temperature variation for HC-PCF based fiber sensor.

Fig. 3 shows the changes of the refection spectra of the sensor with the changes of temperature from 200 to $1200^{\circ} \mathrm{C}$. The spectral envelopes will shift toward longer wavelengths when the temperature is increased. The relation between wavelength shift and temperature keeps good linearity of $99.85 \%$ as depicted in Fig.4. By tracing the resonant peak shift of the envelope, the maximum high temperature sensitivity of the proposed sensor is approximately $15.68 \mathrm{pm} /{ }^{\circ} \mathrm{C}$, which is from 14 to 57 times higher than that of other configuration using similar HC-PCFs but absence of Vernier effect 
(sensitivity of 0.2 to $1.1 \mathrm{pm} /{ }^{\circ} \mathrm{C}$ reported in [18]-[21]), and our result is better than those of compact temperature probes reported in[6], [7], [22], and [23]. Therefore, the proposed novel sensor structure with modified Vernier effect can increase the temperature sensitivity significantly, has a promising prospects in high temperature sensing application.

\section{CONCLUSION}

The proposed novel miniaturized intrinsic fiber Fabry-Perot interferometric sensor (IFFPIs) made by pure silica is capable of withstanding high temperature up to $1200^{\circ} \mathrm{C}$ with stable shape of the interference spectra and good linearity between wavelength shift and temperature change. The sensitivity can be enhanced significantly via the modified Vernier effect, comparing to other configurations using similar HC-PCFs [18]-[21]. The amplification factor can be further increased by reducing the length of the pure silica tip and increasing the length of the HC-PCF cavity. Owing to the advantages of high sensitivity, good stability, compactness and easy fabrication, the proposed sensor has promising prospect in high temperature sensing application.

Acknowledgement: The authors are grateful to the financial support from National Natural Science Foundation of China (no. 61775186); the Fujian Provincial Department of Science and Technology (Project no. 2014H6027); the Marine and Fisheries Bureau of Xiamen (Project no. 16CZB025SF03); and Bureau of Science and Technology of Quanzhou (Project no. 2015G32).

\section{REFERENCES}

[1] J. E. Antonio-Lopez, Z. S. Eznaveh, P. LiKamWa, A. Schülzgen, and R. Amezcua-Correa., "Multicore fiber sensor for high-temperature applications up to $1000^{\circ} \mathrm{C}$," Opt. Lett., 39 (15) 4309, (2014).

[2] Haihu Yu, Ying Wang, Jian Ma, Zhou Zheng, Zhuozhao Luo, and Yu Zheng, "Fabry-Perot Interferometric HighTemperature Sensing Up to $1200^{\circ} \mathrm{C}$ Based on a Silica Glass Photonic Crystal Fiber," Sensors, 18(1), 273, (2018).

[3] P. Zhang et al., "Simplified Hollow-Core Fiber-Based Fabry-Perot Interferometer with Modified Vernier Effect for Highly Sensitive High-Temperature Measurement," IEEE Photonics J., 7(1), 1-10, (2015).

[4] D. Polyzos, J. Mathew, W. N. MacPherson, and R. R. J. Maier., "Effect of Dopant Diffusion on the Long-Term Stability of Fabry-Pérot Optical Fiber Sensors," J. Light. Technol, 35 (24), 5317-5323, (2017).

[5] R. Wang et al., "Fabrication of high-temperature tilted fiber Bragg gratings using a femtosecond laser," Opt. Express, 25(20), 23684, (2017).

[6] Y. Liu, S. Qu, and Y. Li., "Single microchannel high-temperature fiber sensor by femtosecond laser-induced water breakdown," Opt. Lett, 38(3), 335 (2013).

[7] W. Ding, Y. Jiang, R. Gao, and Y. Liu., "High-temperature fiber-optic Fabry-Perot interferometric sensors," Rev. Sci. Instrum., 86 (5), 055001, (2015).

[8] P. Xu et al., " $1200^{\circ} \mathrm{C}$ high-temperature distributed optical fiber sensing using Brillouin optical time domain analysis," Appl. Opt, 55 (21), 5471, (2016).

[9] T. Zhu, T. Ke, Y. Rao, and K. S. Chiang., "Fabry-Perot optical fiber tip sensor for high temperature measurement," Opt. Commun, 283(19), 3683-3685, (2010).

[10] L.-Y. Shao et al., "Sensitivity-enhanced temperature sensor with cascaded fiber optic Sagnac interferometers based on Vernier-effect," Opt. Commun., 336, 73-76, (2015).

[11] P. Zhang et al., "Cascaded fiber-optic Fabry-Perot interferometers with Vernier effect for highly sensitive measurement of axial strain and magnetic field," Opt. Express, 22(16), 19581, (2014).

[12] H. Liao et al., "Sensitivity amplification of fiber-optic in-line Mach-Zehnder Interferometer sensors with modified Vernier-effect," Opt. Express, 25(22), 26898, (2017).

[13] B. Wu, C. Zhao, B. Xu, and Y. Li., "Optical fiber hydrogen sensor with single Sagnac interferometer loop based on vernier effect," Sens. Actuators B Chem, 255, 3011-3016, (2018).

[14] Y. Yang et al., "Sensitivity-enhanced temperature sensor by hybrid cascaded configuration of a Sagnac loop and a F-P cavity," Opt. Express, 25 (26) 33290, (2017).

[15] Y. Zhao, P. Wang, R. Lv, and X. Liu., "Highly Sensitive Airflow Sensor Based on Fabry-Perot Interferometer and Vernier Effect," J. Light. Technol, 34(23), 5351-5356, (2016). 
[16] M. Quan, J. Tian, and Y. Yao., "Ultra-high sensitivity Fabry-Perot interferometer gas refractive index fiber sensor based on photonic crystal fiber and Vernier effect," Opt. Lett, 40(21), 4891, (2015).

[17] D. J. J. Hu et al., "Novel Miniaturized Fabry-Perot Refractometer Based on a Simplified Hollow-Core Fiber with a Hollow Silica Sphere Tip,” IEEE Sens. J, 12(5),1239-1245, (2012).

[18] Y. Wang, D. N. Wang, C. R. Liao, T. Hu, J. Guo, and H. Wei., "Temperature-insensitive refractive index sensing by use of micro Fabry-Perot cavity based on simplified hollow-core photonic crystal fiber," Opt. Lett, 38(3), 269271, (2013).

[19] Y. Yu, X. Chen, Q. Huang, C. Du, S. Ruan, and H. Wei., "Enhancing the pressure sensitivity of a Fabry-Perot interferometer using a simplified hollow-core photonic crystal fiber with a microchannel," Appl. Phys. B, 120 (3) 461-467, (2015).

[20] S. Liu, N. Liu, M. Hou, J. Guo, Z. Li, and P. Lu., "Direction-independent fiber inclinometer based on simplified hollow core photonic crystal fiber," Opt. Lett, 38 (4), 449,(2013).

[21] Z. Wu, Y. Liu, Z. Wang, T. Han, and P. P. Shum, "Temperature-independent strain sensor based on simplified hollow-core photonic crystal fibers,"Proc.PGC, Singapore, 1-2,(2012).

[22] L. V. Nguyen, S. C. Warren-Smith, H. Ebendorff-Heidepriem, and T. M. Monro., "Interferometric high temperature sensor using suspended-core optical fibers," Opt. Express, 24(8),8967, (2016).

[23] P. Wang et al., "Fiber-tip high-temperature sensor based on multimode interference," Opt. Lett, 38(22), 4617, (2013).

[24] F. Gérôme, R. Jamier, J.-L. Auguste, G. Humbert, and J.-M. Blondy., "Simplified hollow-core photonic crystal fiber," Opt. Lett, 35 (8),1157,(2010).

[25] Y.-J. Rao, M. Deng, D.-W. Duan, and T. Zhu., "In-line fiber Fabry-Perot refractive-index tip sensor based on endlessly photonic crystal fiber," Sens. Actuators Phys, 148(1), 33-38, (2008). 